\title{
Periodismo emprendedor. Estrategias para incentivar el autoempleo periodístico como modelo de negocio
}

\author{
Andreu CASERO-RIPOLLÉS \\ Universitat Jaume I (UJI) \\ casero@uji.es \\ Cristina Cullell-MarCh \\ Universitat Jaume I (UJI) \\ cullell@uji.es
}

Recibido: $17 / 10 / 2012$

Aceptado: 23/01/2013

\section{Resumen}

El periodismo está en un escenario de reconversión. El impacto de la crisis ha deteriorado las condiciones y expectativas laborales de sus profesionales. En este marco, el emprendimiento se configura como una nueva opción. Los objetivos de este artículo son el análisis de las bases económicas de la industria periodística y de la incidencia del espíritu emprendedor entre del alumnado universitario, por un lado, y el diseño de metodologías docentes para incentivar el emprendimiento periodístico, por otro. La metodología combina el análisis de datos secundarios y la encuesta cuantitativa $(\mathrm{N}=1077)$. Los resultados sugieren el declive del modelo de negocio tradicional y la escasa orientación hacia el autoempleo del alumnado. El periodismo emprendedor emerge, así, como salida profesional alternativa. No obstante, para posibilitarlo debe potenciarse desde la formación universitaria.

Palabras clave: empresa periodística, emprendimiento, modelo de negocio

\section{Entrepreneurial Journalism. Strategies to Promote Self-Employment in Journalism as a New Business Model}

\begin{abstract}
Journalism is under restructure. The economic crisis affected journalists' working conditions and expectations. In this context a new option arises: the entrepreneurship. The goals of this paper consist of an economic analysis of news companies and the evaluation of the entrepreneurial attitude among university students. It also designs training methods to encourage journalistic entrepreneurship. The methodogy combines data analysis and quantitative survey approach. The paper identifies a decline of traditional business model and a weak student's entrepreneurial attitude. Entrepreneurial journalism emerges as professional option but university education must encourage it.
\end{abstract}

Keywords: news company, entrepreneurship, business model

\section{Referencia normalizada}

CASERO-RIPOLLÉS, Andreu y CULLELL-MARCH, Cristina (2013): "Periodismo emprendedor. Estrategias para incentivar el autoempleo periodístico como modelo de negocio". Estudios sobre el mensaje periodístico. Vol. 19, Núm. especial abril, págs.: 681-690. Madrid, Servicio de Publicaciones de la Universidad Complutense.

Sumario: 1. Introducción 2. Metodología 3. El emprendimiento periodístico; 3.1. El declive del modelo de negocio tradicional; 3.2. La propensión al emprendimiento entre el alumnado universitario; 3.3. El emprendimiento en la asignatura "Empresa Periodística". 4. Conclusiones 5. Referencias bibliográficas 


\section{Introducción ${ }^{1}$}

La crisis está siendo especialmente incisiva con los medios de comunicación y, por extensión, con sus profesionales. A fin de garantizar su supervivencia, las empresas periodísticas afrontan la situación a través de la reconversión de sus modelos de negocio. Este desafío no se ha abordado con una mejora de las estructuras informativas y una mayor calidad sino que se ha resuelto con reducción de gastos, despidos masivos de periodistas, cierre de corresponsalías y de servicios diversos.

En este contexto, se hace más necesario que nunca un replanteamiento de las salidas profesionales de los periodistas. Lejos de trabajar en la estructura orgánica de los medios de comunicación, hoy, la aspiración de un periodista puede orientarse a emprender su propio proyecto profesional autónomamente. No hay duda que la sociedad ha necesitado, necesita y necesitará siempre de periodistas. Sus funciones no están en entredicho, al contrario, deben orientarse hacia la creación de nuevas fórmulas empresariales que permitan canalizar, ejecutar y prestar los servicios que la profesión ofrece a la sociedad y que son cruciales para el buen funcionamiento democrático.

La literatura sobre emprendimiento ha clasificado la figura del emprendedor en base a diferentes rasgos, tales como, explotar oportunidades, necesidad de toma de decisiones rápidas en un contexto de incertidumbre, capacidad de trabajo $\mathrm{y}$, a la vez, desarrollo de cualidades de liderazgo y de coordinación de equipos (Audet y Couteret, 2012). El emprendedor está sometido a situaciones que ponen al límite sus capacidades cognitivas, reconoce y persigue oportunidades (Baron, 1998). Su optimismo y tolerancia a la incertidumbre y, sobretodo, su preferencia a modelar su destino constituyen sus cualidades distintivas (Obschonda et al., 2010). Se trata de un tipo de persona creativa que genera un enorme potencial.

Algunos rasgos de la personalidad pueden tener una influencia directa a la hora de determinar la aparición y activación de una actitud de emprendimiento. Éstos son: innovación, personalidad proactiva, eficacia, tolerancia al estrés, autonomía, control y asunción de riesgos (Rauch y Frese, 2007).

La innovación supone que la persona está interesada en nuevas formas de actuación y sucede cuando la práctica social cambia. El emprendedor exitoso es aquél que pone en práctica aquello que mucha gente piensa pero nunca ha expuesto abiertamente (Tuomi, 2006). En segundo lugar, una actitud proactiva ayuda a afrontar problemas, prever las consecuencias de determinadas acciones toda vez que permite orientarse en un entorno de innovación constante. La pro-actividad se contrapone a una actitud reactiva que piensa más en responder que en hacerse preguntas, en cuestionarse acciones que en reproducirlas y en impulsar procesos de cambio (Ares, 2004).

La eficacia es importante para el emprendedor ya que ha de confiar en sus propias capacidades para actuar de forma variada en situaciones de incertidumbre. La tolerancia al estrés también es clave ya que debe tener una gran capacidad de trabajo y, al mismo tiempo, asumir riesgos personales y financieros, motivo por el cual, puede ser

${ }^{1}$ Este trabajo forma parte de las actividades del Grupo de Innovación Educativa (GIE) en Periodismo de la UJI y se integra en el proyecto de I+D con referencia P1-1B2010-53, financiado por la Universitat Jaume I y la Fundación Caixa Castelló-Bancaixa. 
objeto de fuertes presiones e inseguridades. Finalmente, la autonomía e independencia son básicas para evitar las restricciones pre-establecidas.

Sobre las motivaciones que alientan al emprendimiento, no son sólo de tipo economicista y de desarrollo profesional (Pfeilstetter, 2011) sino que también pueden obedecer a vocaciones de tipo social o comunitario (Austin, Stevenson et al., 2006; Defourny y Nyssens, 2010). Este emprendimiento social (Nicholls, 2006), que aporta un beneficio al conjunto de la ciudadanía, resulta de especial interés para el ámbito de la empresa periodística, dada su orientación hacia el servicio público.

Los factores que potencian el emprendimiento son varios. Uno de ellos, es el sistema educativo. Este no sólo tiene que formar personas que puedan observar, describir y analizar la realidad sino que también tiene que poder crear personas con iniciativa, capaces de detectar una necesidad, una oportunidad, innovar y al mismo tiempo asumir la responsabilidad de hacerlo posible a través de acciones concretas que cambien la realidad (Kirby, 2008). Las universidades juegan un papel clave en el desarrollo de actitudes de emprendimiento (Henry et.al, 2005). Hoy se espera que estas instituciones adopten un rol activo, no sólo como creadoras de capital social, sino también cómo impulsoras de la profesionalización y cómo vivero de empresas. En este sentido, son muchas las universidades que han adoptado el emprendimiento y la innovación como ejes de su acción (Politis et al., 2010)

El contexto social y familiar juega un importante papel en el desarrollo de las intenciones de autoempleo. Los entornos familiares que desarrollan actividades empresariales propias acentúan una influencia positiva hacia el emprendimiento (Laspita et al., 2012). Asimismo, las intenciones emprendedoras también pueden verse condicionadas por factores heredados de los progenitores. Los genes pueden afectar el desarrollo de mecanismos cerebrales que fomentan características y actitudes específicas de emprendimiento. La preferencia al autoempleo está condicionada genéticamente y es mayor en las mujeres que en los hombres (Zhang et al., 2009).

Este artículo se plantea tres objetivos. En primer lugar, analizar la situación económica de la industria periodística para fundamentar la necesidad del autoempleo como salida profesional para los periodistas. En segundo término, conocer y calibrar la incidencia del espíritu emprendedor entre los estudiantes universitarios. Finalmente, diseñar una propuesta metodológica para incentivar el autoempleo y el emprendimiento periodísticos.

\section{Metodología}

El diseño metodológico se basa en la combinación de dos técnicas de investigación: el análisis secundario de datos y la encuesta cuantitativa. La primera de ellas permitirá conocer la situación actual del modelo de negocio tradicional de las empresas periodísticas en España. La segunda facilitará calibrar el grado de propensión y valoración del emprendimiento entre el alumnado universitario.

La muestra del análisis secundario de datos está integrada por las empresas editoras de diarios españolas y el período de referencia estudiado es el comprendido entre los años 2007 y 2010. Se consideran tres variables: ingresos por venta de ejemplares, ingresos publicitarios y beneficios empresariales. Por su parte, la muestra de la en- 
cuesta cuantitativa está conformada por los estudiantes universitarios de primer año del curso 2009-2010 de la Universitat Jaume I de Castellón ( $\mathrm{N}=1077)$. El cuestionario está compuesto por preguntas cerradas de estimación basadas en una escala de 1 (mínimo) a 7 (máximo). Las variables consideradas son la percepción del emprendimiento, los factores que lo favorecen y los rasgos del alumnado que se asocian al mismo.

\section{El emprendimiento periodístico}

\subsection{El declive del modelo de negocio tradicional}

En los últimos años, la industria periodística tradicional ha sufrido un fuerte deterioro en España. Uno de los ejemplos más claros de este proceso lo representan las empresas editoras de diarios. El impacto de la crisis económica internacional, iniciada en 2008, ha tenido efectos devastadores sobre el sector (Larrañaga, 2009; Díaz Nosty, 2011; Guallar, 2011). La totalidad de las fuentes de financiación de la prensa se han visto afectadas por la recesión.

En su fórmula clásica, los diarios han basado su obtención de ingresos económicos en una doble financiación procedente, por un lado, de la venta del producto a los lectores y del recurso a la publicidad, por otro (Sonnac, 2009). Ambas vías han notado las consecuencias de la crisis. En el caso de la venta de ejemplares, que incluye también las suscripciones, los periódicos españoles perdieron un $14,2 \%$ de su volumen de negocio entre 2007 y 2010. Aún, así, el total de facturación por este concepto en 2010 ascendió a 1.115,9 millones de euros. Por su parte, los ingresos publicitarios cayeron un $42,9 \%$ en el período indicado. Una cifra que provocó que la facturación por publicidad pasara de 1.461 millones de euros en 2007 a 834,5 millones de euros en 2010.

Estos datos demuestran el impacto negativo de la recesión económica internacional en la industria periodística. No obstante, a la situación actual de fuerte deterioro de sus bases financieras también han contribuidos dos procesos previos que han jugado un papel fundamental. El primero es la crisis estructural que vive la prensa desde mediados de la década de los 90 (Casero-Ripollés, 2010). Esta dinámica que ha favorecido un lento pero progresivo desgaste del sector se debe a factores como el alejamiento del público joven que está abandonando la lectura de diarios (Casero-Ripollés, 2012), el aumento del consumo de noticias online o la pérdida de influencia de la prensa impresa frente a otros medios informativos como la televisión o Internet, entre otros. El segundo proceso tiene que ver con la innovación tecnológica, asociada a la digitalización y la convergencia, que está transformando el periodismo (Díaz-Noci, 2010).

Con todo, la industria periodística atraviesa un momento de extrema gravedad en términos económicos. Las consecuencias de ello son diversas, pero se pueden concretar en cuatro puntos que dibujan un cuadro dantesco. El primero es la drástica caída de beneficios experimentada por las empresas periodísticas españolas que, entre 2007 y 2010 , han perdido un $66,25 \%$ de su Ebitda, que mide el beneficio bruto de explotación. El segundo se ha traducido en despidos masivos de profesionales de los medios periodísticos que, según la Federación de Asociaciones de Periodistas de España, supera las 7.800 personas desde el inicio de la crisis. El tercero es el aumento de la precariedad laboral de los periodistas que están viendo como se deterioran sus condiciones de trabajo. Como ejemplo de ello, una empresa digital retribuía cada no- 
ticia de 800 caracteres con 0,75 euros. Algo que motivó la puesta en marcha de la campaña \#gratisnotrabajo por parte de las asociaciones profesionales de periodistas para denunciar la situación. Finalmente, en cuarto lugar, se ha producido el cierre de medios, incluso de ámbito nacional, como los gratuitos $A D N$, Metro y Qué, el digital Soitu o el periódico de información general Público.

Esta situación se agrava, aún más si cabe, por la ausencia de un nuevo modelo de negocio para el periodismo adaptado al entorno digital (Curran, 2010). Los diarios no consiguen encontrar una formula adecuada para generar beneficios económicos suficientes de su actividad informativa a través de Internet (Casero-Ripollés, 2010). Todo ello pese a que el número de lectores online no deja de aumentar, ya que en España, según datos de Eurostat, el porcentaje de población que consume noticias en la Red ha pasado representar el 24\% en 2007 a suponer el 45\% del total en 2011.

El deterioro de las bases tradicionales de la industria periodística y la ausencia de un nuevo modelo adaptado al entorno digital plantea la necesidad de explorar nuevas vías para el negocio periodístico (Campos-Freire, 2010). Una de ellas es el emprendimiento que se alza como una nueva opción para fomentar el trabajo y el autoempleo en el ámbito del periodismo. La puesta en marcha de proyectos personales y a pequeña escala por parte de los propios periodistas se configura en el escenario actual como una alternativa factible y real. No obstante, para que pueda hacerse realidad resulta indispensable introducir, y estimular, el emprendimiento en la formación de los periodistas.

\subsection{La propensión al emprendimiento entre el alumnado universitario}

Para conocer el grado de propensión y valoración del emprendimiento entre los futuros profesionales se toman como referencia los datos de un estudio de la Cátedra INCREA y el Foro Jovellanos de la Universitat Jaume I de Castellón (UJI). Este trabajo, basado en la aplicación de la técnica de la encuesta cuantitativa a una muestra de los estudiantes universitarios de primer año del curso 2009-2010 ( $\mathrm{N}=1077$ ), pretende generar indicadores sobre el emprendimiento. A partir de los mismos, podemos observar tres variables claves para conocer la extensión del emprendimiento entre el alumnado universitario: la percepción del emprendimiento, los factores que lo favorecen y los rasgos que se asocian al mismo.

En relación a las percepciones hacia el emprendimiento, se observa que los estudiantes de la UJI creen mayoritariamente que trabajarán como asalariados una vez finalicen los estudios y ven poco probable crear su propia empresa, en 5.08 (siendo 1 poco y 7 mucho). Existe, como punto de partida, un interés reducido hacia el emprendimiento por parte del alumnado.

Por otro lado, entre los factores que favorecen las intenciones de emprendimiento se observa que los condicionamientos familiares a la hora de crear la empresa son de relevancia media (3.66), teniendo un mayor ascendente la opinión de las amistades (3.83).

Finalmente, entre los rasgos del alumnado que se asocian a la figura del emprendedor, observamos como los estudiantes de la UJI tienen una capacidad de asunción de riesgos elevada así como de innovación y de producción de nuevas ideas, situándose ambas en un 5.09, uno de los porcentajes más altos de todas las variables. Por otro 
lado, la propensión del alumnado para soportar situaciones de incertidumbre es moderadamente elevada (4.79).

Tabla 1. Actitudes del alumnado de la UJI ante el emprendimiento.

Fuente: Cátedra INCREA - UJI

\begin{tabular}{|c|c|c|}
\hline Variable & Pregunta & $\begin{array}{c}\text { Media } \\
(1-7)\end{array}$ \\
\hline \multirow{2}{*}{$\begin{array}{l}\text { Percepción de em- } \\
\text { prendimiento }\end{array}$} & $\begin{array}{l}\text { Indica con qué probabilidad crees que trabajarás como asalariado una vez } \\
\text { acabes tus estudios }\end{array}$ & 5.08 \\
\hline & $\begin{array}{l}\text { Indica con qué probabilidad crearás tu propia empresa una vez acabes tus } \\
\text { estudios o durante los mismos }\end{array}$ & 3.10 \\
\hline \multirow{2}{*}{$\begin{array}{l}\text { Factores que favore- } \\
\text { cen las intenciones } \\
\text { de emprendimiento }\end{array}$} & $\begin{array}{l}\text { En qué medida crees que tus familiares más cercanos opinan que deberías } \\
\text { crear tu propia empresa }\end{array}$ & 3.66 \\
\hline & $\begin{array}{l}\text { En qué medida crees que tus mejores amigos opinan que deberías crear tu } \\
\text { propia empresa }\end{array}$ & 3.83 \\
\hline \multirow{3}{*}{$\begin{array}{l}\text { Rasgos del alum- } \\
\text { nado que se asocian } \\
\text { el emprendimiento }\end{array}$} & Soy capaz de producir nuevas ideas & 5.09 \\
\hline & Soy capaz de asumir riesgos & 5.09 \\
\hline & Soy capaz de soportar situaciones de incertidumbre & 4.79 \\
\hline
\end{tabular}

En base a los datos, se puede sostener que el alumnado de la UJI comparte ciertos rasgos que se pueden identificar con el emprendedor. No obstante, su orientación al autoempleo y a la creación de nuevas empresas es muy baja (3.10), puesto que la mayor parte que cree que trabajará como asalariado una vez finalice los estudios. Una percepción que, a tenor del deterioro del modelo negocio tradicional, es poco acorde con la realidad que vive el mundo del periodismo.

En este contexto, marcado por la crisis del sector y la escasa propensión al autoempleo de los futuros profesionales, resulta fundamental incorporar el emprendimiento como un elemento clave en la formación de los periodistas. La apuesta por el emprendimiento constituye hoy una vía totalmente válida para la inserción laboral de los periodistas. Las tecnologías digitales han reducido las barreras de entrada para impulsar proyectos periodísticos y los cambios en el entorno comunicativo hacen más pertinente que nunca la innovación y la creatividad para propiciar el surgimiento de nuevas iniciativas y servicios periodísticos.

\subsection{El emprendimiento en la asignatura "Empresa Periodística"}

La asignatura "Empresa Periodística" resulta especialmente adecuada para la incorporación del emprendimiento como competencia docente y formativa. Ello requiere el diseño de metodologías y estrategias docentes para incentivar y motivar al alumnado hacia el autoempleo.

En este marco, es clave potenciar las competencias de emprendimiento, autonomía profesional, liderazgo, iniciativa y toma de decisiones. Especialmente teniendo en cuenta que, en la UJI, ésta es una asignatura del segundo semestre del tercer curso y, por lo tanto, se sitúa en el tramo final de la formación del alumnado. 
El objetivo de aprender a emprender se ha fijado como uno de los principales de "Empresa Periodística". Por ello la elaboración de un proyecto profesional propio deviene un objetivo fundamental. Su diseño, desarrollo y ejecución aumenta sustancialmente la implicación y la motivación del alumnado hacia la asignatura (Harmeling, 2011).

Para llevar a cabo dicho proyecto, el alumnado tiene que detectar unas necesidades sociales y en base a ellas diseñar y, si cabe, ejecutar un servicio a la comunidad. El profesorado debe guiar, ayudar y asistir al estudiantado para que adquiera verdaderas actitudes y habilidades de emprendimiento (Carrier, 2008).

En este marco se emplea como metodología docente el aprendizaje de servicio. Se trata de una propuesta de trabajo cooperativo y colaborativo donde el estudiante desarrolla competencias orientadas a mejorar su comprensión de la realidad social, económica, cultural, etc. que afecta a los miembros de una comunidad y desenvuelve una clara voluntad de transformación social (Martínez, 2008). La introducción de esta metodología en la asignatura "Empresa Periodística" es apropiada por tres motivos. En primer lugar, porque se produce una combinación continua entre teoría y práctica y al mismo tiempo también se introducen procesos reflexivos en nivel individual y en grupo para mejorar la práctica. En segundo término, porque es a través de la práctica cuando se entienden mejor los contenidos curriculares. Y, finalmente, porque el aprendizaje que se produce tiene una función directa sobre la comunidad. Las propuestas de aprendizaje de servicio aportan nuevas reflexiones, sensibilidades y conocimientos que generan nuevas relaciones y compromisos personales, cívicos, éticos y sociales (Campo, 2008). Una cuestión que el periodismo debe aprovechar para el efectivo cumplimiento de su responsabilidad pública.

Entre los proyectos periodísticos elaborados por el alumnado de "Empresa Periodística" y basados en el aprendizaje de servicio destaca el proyecto AidPoint que pretende facilitar la comunicación entre los enfermos de cáncer de la Comunidad Valenciana; o Notelodiranenclase $e^{2}$ un portal informativo destinado al alumnado de la UJI.

\section{Conclusiones}

El periodismo está inmerso en un complejo escenario de redefinición. La crisis económica está afectando extraordinariamente a las empresas periodísticas y, por extensión, a sus profesionales. El modelo vigente en el que se había asentado la industria periodística se tambalea y da muestras de agotamiento. En este contexto, la reformulación del modelo de negocio ha pasado al primer plano del debate sobre el futuro del periodismo. La búsqueda de nuevas fórmulas para canalizar, ejecutar y prestar sus servicios se ha convertido en una necesidad urgente, más si consideramos el carácter indispensable del periodismo en la actual sociedad-red. Junto a estos intentos, se abre para los profesionales del periodismo un nuevo escenario de oportunidades para poner en práctica iniciativas de autoempleo gracias a las tecnologías digitales. Estas redu-

${ }^{2}$ http://notelodiranenclase.wordpress.com/page/2/ 
cen las barreras de entrada al sector y hacen factible el desarrollo del espíritu emprendedor entre los periodistas como vía alternativa para el ejercicio de la profesión. El periodismo emprendedor se puede configurar como una salida laboral innovadora clave a corto y medio plazo.

Los resultados observados constatan que la cultura estudiantil es poco proclive a emprender nuevos proyectos profesionales y avanzar hacia el autoempleo. Por ello, es necesario elaborar propuestas formativas que potencien las competencias y mecanismos para promocionar el espíritu emprendedor como opción profesional entre los futuros periodistas.

La incorporación del emprendimiento como competencia docente es especialmente apropiada en el ámbito de la "Empresa Periodística". Ésta debe fundamentarse en la metodología del aprendizaje de servicio aplicado a la realización de proyectos empresariales por parte del propio alumnado que respondan a necesidades sociales. Esta fórmula constituye la mejor vía para formar a los periodistas emprendedores del futuro.

\section{Referencias bibliográficas}

ARES, Antonio (2004): "La conducta proactiva de los emprendedores". Portularia, 4: 493-498.

AUDET, Josée y COUTERET, Paul (2012): "Coaching the entrepreneur: features and success factors". Journal of Small Business and Enterprise Development 19: 515531.

AUSTIN, James; STEVENSON; Howard y WEU-SKILLERN, Jane (2006): "Social and Commercial Entrepeneurship: Same, Different or Both?"Estrepeneurship Theory and Practice, 30: 1-22.

BARON, Robert (1998): “Cognitive mechanisms and entrepreneurship. Why entrepreneurs think differently?” Journal of Business Venturing, 13: 275-294.

CAMPO, Laura (2008): "El aprendizaje servicio en la universidad como propuesta pedagógica". En MARTÍNEZ, M. (ed.): Aprendizaje de Servicio y Responsabilidad Social de las Universidades. Madrid, Ministerio de Educación y Ciencia.

CAMPOS-FREIRE, Francisco (2010): "Los nuevos modelos de gestión de las empresas mediáticas". Estudios sobre el mensaje periodístico, 16: 13-30. Madrid, Servicio de Publicaciones de la Universidad Complutense.

CARRIER, Camille (2008): "Strategies for teaching entrepreneurship what else beyound lectures, cas estudies and business plans". En Fayolle, A. (ed.) Handbook of research in entrepreneurship education. Cheltenham, Edward Elgar.

CASERO-RIPOLLÉS, Andreu (2012): "Beyond Newspapers: News Consumption among Young People in the Digital Era". Comunicar, 39: 151-158.

CASERO-RIPOLLÉS, Andreu (2010): "Prensa en Internet: nuevos modelos de negocio en el escenario de la convergencia". El profesional de la información, 19 (6): 595-601. 
CURRAN, James (2010): “The Future of Journalism”. Journalism Studies, 11 (4): 464-476.

HENRY, Cloette; HILL, Frances y LEITCH, C. (2005): "Entrepreneurship education and training: can entrepreneurship be taught?". Education + Training, 47: 98 111.

DEFOURNY, Jacques y NYSSENS, Marthe (2010): “Conceptions of Social Enterprise and Social Entrepreneurship in Europe andt he United States: Convergences and Divergences". Journal of Social Entrepreneurship, 1: 32-53.

DÍAZ-NOCI, Javier (2010): "Medios de comunicación en Internet: algunas tendencias". El profesional de la información, 19 (6): 561-567.

DÍAZ NOSTY, Bernardo (2011): Libro negro del periodismo en España. Madrid, APM.

GUALLAR, Javier (2011): "Prensa digital en 2010". AnuarioThinkEPI, 5: 101-105.

HARMELING, Susan (2011): "Re-storying an entrepreneurial identity: education, experience and self-narrative". Education + Training,53: $741-749$.

KIRBY, David (2008):“"Changing the entrepeneurship education paradigm”. En Fayo1le, A. (ed.) Handbook of research in entrepreneurship education. Cheltenham, Edward Elgar.

LASPITA, Stravroula y BREGUST, Nicola (2012): "Intergenerational transmission of entrepreneurial intentions". Journal of Business Venturing, 27:414-435.

LARRAÑAGA, Julio (2009): "La crisis del modelo económico de la industria de los periódicos". Estudios sobre el Mensaje Periodístico, 15: 61-80. Servicio de Publicaciones de la Universidad Complutense.

MARTÍNEZ, Miquel (2008): Aprendizaje de Servicio y Responsabilidad Social de las Universidades. Madrid, Ministerio de Educación y Ciencia.

NICHOLS, Alex (2006): Social Entrepreneurship: New Models of Sustainable Social Change. Oxford, Oxford University Press.

OBSCHONDA, Martin; SILBEREISEN, Rainer y SCMITT, Eva (2010): "Entrepreneurial intention as developmental outcome". Journal of Vocational Behavior, 77: 63-72.

POLITIS, Diamanto; WINBORG, Joakim y LINDHOLM, Asa (2010): "Exploring the resource logic of student entrepreneurs". International Small Business Journal, 30: 659-683.

PFEILSTETTER, Richard (2011): “El emprendedor. Una reflexión crítica sobre usos y significados actuales del concepto". Gazeta de Antropología, 27: 54-67.

RAUCH, Andreas y FRESE, Michael (2007): "Let's put the person back into entrepreneurship research: A meta-analysis on the relationship between business owners' personality traits, business creation, and success". European Journal of Work and Organizational Psychology, 16, 353-385. 
SONNAC, Nathalie (2009): "L'économie de la presse: vers un nouveau modèle d'affaires." Les cahiers du journalisme, 20: 22-43.

TUOMI, Ikka (2006): Networks of Innovation. Change and Meaning in the Age of Internet. Oxford, Oxford University Press.

ZHANG, Zhen; ZYPHUER, Michael; NARAYAN, Jayanth; ARVEY, Richard (2009): "The genetic basis of entrepreneurship: effects of gender and personality". Organizational Behavior and Human Decision Processes, 110: 93-107.

\section{Andreu CASERO-RIPOLLÉS}

Universitat Jaume I de Castellón (UJI)

Facultad de Ciencias Humanas y Sociales. Departamento de Ciencias de la Comunicación Profesor Titular y vicedecano - director del Grado en Periodismo

casero@uji.es

\section{Cristina CULLELL-MARCH}

Universitat Jaume I de Castellón (UJI)

Facultad de Ciencias Humanas y Sociales. Departamento de Ciencias de la Comunicación Profesora de Empresa periodística.

cullell@uji.es 